\title{
Controlling adsorbate vibrational lifetimes using superlattices
}

\author{
Steven P. Lewis \\ Department of Physics and Astronomy and Center for Simulational Physics, \\ University of Georgia, Athens, GA 30602-2451. \\ Andrew M. Rappe \\ Department of Chemistry and Laboratory for Research on the Structure of Matter, \\ University of Pennsylvania, Philadelphia, PA 19104.
}

(October 25, 2018)

\begin{abstract}
We propose using short-period superlattices as substrates to control the vibrational relaxation dynamics of adsorbate overlayers. The mass modulation of superlattices creates both band gaps and large spectral enhancements in the phonon density of states. These modifications can dramatically alter the coupling between vibrational modes of the adsorbate overlayer and the substrate lattice, thereby significantly affecting the lifetime of adsorbate modes.
\end{abstract}

\section{INTRODUCTION}

The lowest energy vibrational excitations of molecules adsorbed on metal surfaces generally correspond to motion of the molecule as a whole about the adsorption bond, i.e., frustrated translations and rotations. These modes play an important role in thermally activated processes at surfaces, such as catalysis and surface diffusion. [1] It would, therefore, be highly desirable to be able to control the dynamical behavior of these vibrations. We propose a way to dramatically alter the lifetime of low-frequency adsorbate modes by suitably modifying the composition of the substrate.

We recently developed a theory for the relaxation dynamics of adsorbate vibrational modes whose frequency lies within the long-wavelength acoustic part of the substrate phonon spectrum. [2 5 This frequency range is relevant, for example, for in-plane frustrated translations (FT) of molecules on metal surfaces. We have shown that the relaxation of these modes is governed by resonant coupling to bulk substrate phonons; i.e., the vibrating overlayer radiates its energy into resonant acoustic waves. [6] All other relaxation channels, such as substrate electron-hole pair excitation, are typically sub-dominant. This result suggests that the lifetime of FT and other low-lying adsorbate modes can be significantly altered by changing the substrate phonon spectrum at the resonant frequency.

In this paper we explore the use of superlattices to achieve this goal. For the last two decades, superlattices have received considerable attention by materials researchers because of their unusual and highly tunable properties. Superlattices consist of a periodic alternation of layers of two or more different materials. For simplicity, this paper focuses on superlattices of two materials, $A$ and $B$. The resulting periodic mass modulation causes frequency gaps to open in the phonon spectrum for modes propagating parallel to the stacking direction, $\hat{s}$. The size and placement of the band gaps are determined by compositional parameters of the superlattice, such as the mass ratio of materials $A$ and $B$ and the modulation period. The modes expelled from band-gap regions gather near van Hove singularities at the gap edges. Thus, the superlattice mass modulation enhances the bulk phonon spectrum at some frequencies while depleting it at others.

We consider an ordered adsorbate overlayer on the surface of such a superlattice, with the surface normal aligned along $\hat{s}$. A $\vec{k}_{\|}=0 \mathrm{FT}$ excitation of the overlayer will only couple to bulk modes propagating parallel to $\hat{s}$. Thus, depending on the superlattice compositional parameters, the FT frequency may fall in a region of either depleted or enhanced phonon density of states (DOS), compared to the pure substrate. In the former case, resonant phonon emission is eliminated as a FT decay channel, because there are no phonon states at the resonant frequency. In the latter case, one might expect enhanced coupling because of the increased phase space. However, before reaching that conclusion, it is necessary to consider the coupling strength to modes in the van Hove singularities. In this work, we examine all of these issues using a model system corresponding to an ordered adsorbate overlayer on a face-centered cubic (fcc) superlattice.

Acoustic waves in superlattice substrates have been investigated previously. 17 11] In particular, Camley et al. derived vibrational dispersion relations for both infinite and semi-infinite superlattices in the elastic-continuum limit (i.e., both the solid as a whole and the $A$ and $B$ layers individually are treated as continuous media). [9] They 
focused on superlattices with a modulation wavelength of $1000 \AA$ or more. In this paper, we opt for an atomistic approach instead of an elastic-continuum model, because we are interested in superlattices of small enough modulation wavelength that variations by even a single atomic layer cause noticeable changes in the bulk phonon spectrum. Nevertheless, the basic physical picture presented in Ref. [9] still applies.

Figure 6 of Ref. [9] is a plot of $\omega$ vs. $k_{\|}$for an infinite superlattice. This plot shows that the mass modulation creates frequency gaps in the bulk vibrational spectrum not only at $k_{\|}=0$, but also at finite $k_{\|}$. Indeed, for any frequency, there will be regions of the $k_{\|}$-plane for which there are no propagating acoustic modes and other regions for which propagating acoustic modes exist. Points on the boundary between these two types of regions are van Hove singularities. This picture is very encouraging, since $k_{\|}$-resolved experimental surface techniques, such as inelastic helium-atom scattering, [12] could then be used to exhibit the whole range of behaviors predicted here for adsorbate vibrations coupled to a superlattice. Camley et al., also present $\omega$ vs. $k_{\|}$for a semi-infinite superlattice (Fig. 8 of Ref. [9]). They show that a band of surface waves appears in each bulk band-gap region.

\section{MODEL}

We use lattice dynamics to study the vibrational coupling between the adsorbate overlayer and the superlattice substrate. In this approach, the atoms are treated as point masses, and each atomic degree of freedom of the system is coupled harmonically to all others. The normal modes of vibration are obtained by solving the generalized eigenvalue problem, $\mathbf{K} \cdot x=-\omega^{2} \mathbf{M} \cdot x$, where the eigenvector $x$ resides in the $3 J$-dimensional configuration space of the $J$ atoms, with origin at the equilibrium configuration, the eigenvalue $\omega^{2}$ is the square of the normal-mode frequency, $\mathbf{M}$ is the diagonal $3 J \times 3 J$ matrix of atomic masses, and $\mathbf{K}$ is the symmetric $3 J \times 3 J$ matrix of harmonic force constants. For metals and non-polar semiconductors and insulators, a cutoff can be defined so that only coupling constants above a given magnitude are included. This makes the $\mathbf{K}$ matrix sparse.

Our model substrate is an fcc superlattice with the stacking axis and the surface normal both parallel to the [001] crystallographic axis. Integers $N_{A}$ and $N_{B}$ represent the number of atomic (001) planes of materials $A$ and $B$, respectively, in each superlattice period $\left(N=N_{A}+N_{B}\right)$. The overall thickness of the substrate in our calculations is $250 N$. Our model overlayer consists of an ordered array of diatomic molecules forming a $c(2 \times 2)$ pattern (i.e., checkerboard pattern). In-plane periodic boundary conditions are used, making the model system of infinite extent parallel to the surfaces and superlattice interfaces, but restricting our analysis to $k_{\|}=0$ modes.

For simplicity in this exploratory research, we make the approximation that the force constants coupling the substrate atoms are insensitive to the chemical species. Therefore, varying the superlattice composition is achieved simply by altering the mass matrix $\mathbf{M}$. For real systems, the mass modulation accounts for the majority of the effect, with compositionally varying force constants contributing essentially no new physics.

The major conclusions of this research are insensitive to the specific details of the model system. However, we study this particular structural model for two important reasons. First, this structure is often experimentally realized for diatomic adsorbates on fcc-metal (001) substrates. Second, this choice of model allows us to use the force constants we obtained from earlier density-functional theory calculations for the prototypical system of $\mathrm{CO}$ on $\mathrm{Cu}(001)$. [2,5] The vibrational frequencies computed in that study are in excellent quantitative agreement with experiment. See Ref. [5] for numerical values of the force constants, as well as details of how they were computed and comparisons to experiment. For definiteness and ease of comparison to our earlier work, we also choose the masses of $\mathrm{CO}$ and $\mathrm{Cu}$ for the adsorbate and $A$-atom masses, respectively.

Given the model described above, there remain three parameters for specifying the system: the mass ratio $m_{B} / m_{A}$ of the two superlattice materials and the layer thicknesses, $N_{A}$ and $N_{B}$. Experience shows that the mass ratio needs to be fairly large in order to create sizable phonon band gaps. We have chosen a mass ratio of 3:1 (e.g., Au and $\mathrm{Cu}$ have this mass ratio).

The modulation period $N$ should be chosen so that the FT frequency is near or within a phonon band gap. If the frequency of resonant phonons in the pure material corresponds to a wavelength spanning about $M$ atomic layers, then Bragg theory suggests that a modulation period of $N=M / 2$ layers will open a band gap at or near the resonant frequency. For the model parameters corresponding to $c(2 \times 2)-\mathrm{CO}$ on $\mathrm{Cu}(001)$, our base system, this condition gives a modulation wavelength of about $N=8$ layers. This value is to be contrasted with the elastic-continuum work of Ref. [9], which considered a superlattice with $1500 \AA$ modulation wavelength, corresponding to $N \approx 800$ layers. The resulting lowest band gap was $0.05 \mathrm{~cm}^{-1}$ wide, centered at a frequency of $0.24 \mathrm{~cm}^{-1}$. These values are two orders of magnitude too small for our purposes, and we thus need to consider a superlattice modulation that is two orders of magnitude narrower. The distribution of the $N=8$ layers between materials $A$ and $B$ is left as a variable parameter. 


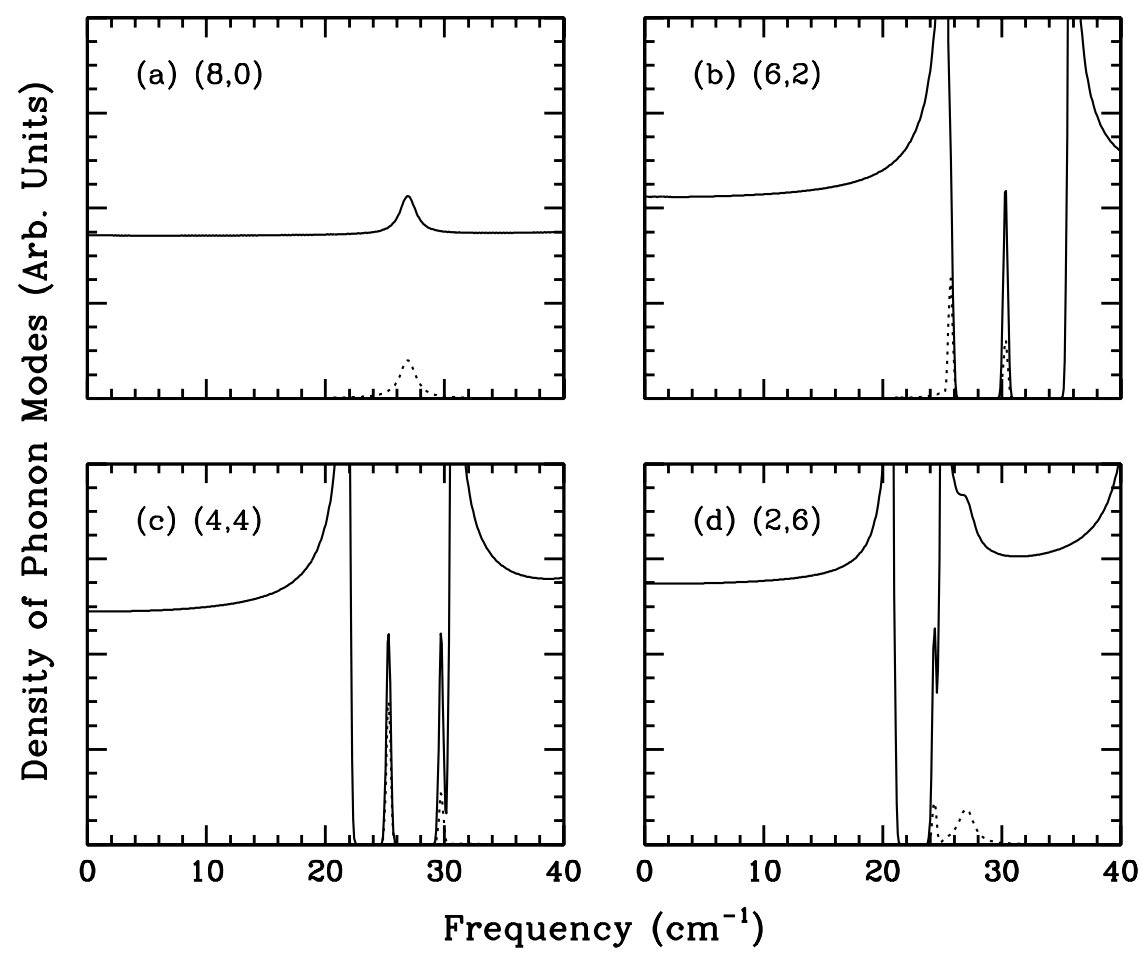

FIG. 1. Vibrational spectrum for an adsorbed superlattice substrate with $N=8$. Each panel represents a different partitioning of the superlattice period into $A$ and $B$ layers. In terms of the symbol $\left(N_{A}, N_{B}\right)$ described in the text: (a) $(8,0)$; (b) $(6,2) ;($ c) $(4,4) ;(d)(2,6)$. The solid curve in each panel gives the full vibrational density of states, whereas the dashed curve gives the density of states projected onto the frustrated-translational mode.

\section{RESULTS}

Figure 1(a)-(d) shows the low-frequency portion of the vibrational DOS of the coupled adsorbate/substrate system for $N=8$ superlattices. The dashed curve in each panel is the DOS projected onto the idealized FT coordinate. Each panel corresponds to a different partitioning of the 8 layers of the superlattice period into $A$ and $B$ layers. The symbol $\left(N_{A}, N_{B}\right)$ in the figure panels specifies the partitioning. The $(8,0)$ panel (Fig. I $\left.1(\mathrm{a})\right)$ corresponds to a pure substrate of material $A$. For the present choice of masses and force constants, this system is identical to our vibrational spectrum in Refs. [2] and online [5]. The broad peak centered at $27 \mathrm{~cm}^{-1}$ is the FT resonance, whose natural line width corresponds to a lifetime of $3.0 \mathrm{ps}$.

As soon as mass modulation is introduced into the substrate, band gaps open in the DOS near the FT frequency, as predicted. In addition there are band gaps at higher frequencies corresponding to higher order Bragg reflections. The band gaps are accompanied by the large spectral enhancement on either side of the gap characteristic of van Hove singularities. Notice that isolated peaks often appear in the band gaps. These peaks are mixtures of the surface states described by Camley et al. in Ref. [9] with the adsorbate frustrated translation. They are truly $\delta$-functions; the width that appears in the figure results from Gaussian smoothing of the spectra.

For the $(6,2)$ superlattice, the van Hove singularity below the band gap coincides in frequency with the FT mode (see Fig. 1 1 (b)). Despite the large density of bulk phonon states at that frequency, the FT mode does not form a broad resonance. Rather, it remains a narrow, $\delta$-function-like peak. This suggests that the coupling strength of the FT to bulk modes in this peak is very weak. We explore this issue further in the discussion below.

As the content of the more massive material $(B)$ in the superlattice increases, the band gap narrows and shifts to lower frequency. These changes reflect the overall contraction of much of the phonon spectrum due to the $1 / \sqrt{M}$ like dependence of vibrational frequencies. For the $(4,4)$ superlattice, this effect causes the lower edge of the band gap to come below the FT frequency, leaving the adsorbate mode in the gap with no phonons to couple to (see 


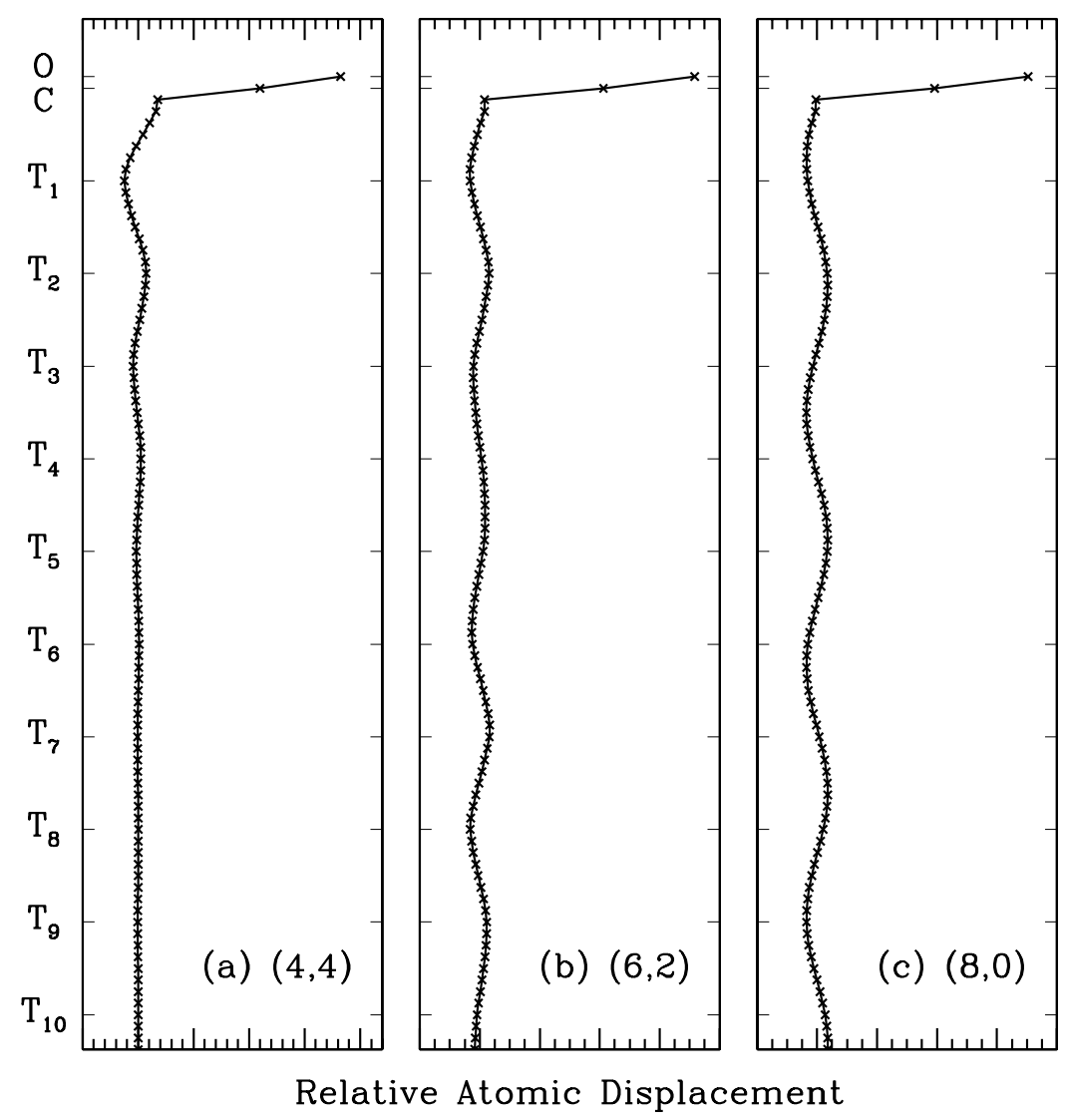

FIG. 2. Atomic displacement patterns for frustrated-translational normal modes. In each panel, the vertical axis denotes the position of a layer of atoms relative to the surface, and the horizontal axis denotes the relative displacement of each layer away from equilibrium for the given normal mode. The symbol $T_{i}$ denotes the interface between the $i$ th and $(i+1)$ th superlattice periods. (a) Isolated FT mode in the $(4,4)$ spectrum. (b) Center of the FT resonance of the $(6,2)$ spectrum. (c) Center of the FT resonance of the $(8,0)$ spectrum.

Fig. 1(c)). This feature is now an infinitely narrow $\delta$-function. Resonant coupling to substrate phonons, therefore, contributes nothing to FT relaxation, leaving previously marginal processes, such as electron-phonon, multi-phonon, and anharmonic coupling, as the dominant decay channels.

For the $(2,6)$ superlattice, the narrowing and lowering of the gap has caused the upper edge of the gap to come below the FT frequency, leaving the FT mode once again well within the bulk phonon continuum. The coupling to these bulk modes is strong enough to produce a broad FT resonance peak, as in the case of the pure $(8,0)$ substrate.

\section{DISCUSSION}

To gain a deeper understanding of the effect of varying superlattice composition on the FT dynamics, we examine, in Fig. 2, the atomic displacement patterns of FT-related normal modes for the $(4,4),(6,2)$, and $(8,0)$ systems. For the $(6,2)$ and $(8,0)$ systems, we display the modes at the center of the FT resonance peak. In each panel, the vertical axis denotes the position of a layer of atoms relative to the surface, and the horizontal axis denotes the relative displacement of each layer away from equilibrium for the given normal mode. For clarity only $\sim 80$ atomic layers of the 2,000-layer substrate are shown.

Figure 2 highlights the characteristic delocalization that occurs when an isolated mode comes into resonance with a continuum of states. The isolated FT mode for the $(4,4)$ system, shown in Fig. 2(a), is exponentially localized at the surface. The side-to-side "wagging" of the CO molecules dominates the displacement pattern, with the amplitude on substrate layers rapidly decaying as a function of penetration distance. In contrast, the resonance modes pictured in Figs. 2(b) and (c) for the $(6,2)$ and $(8,0)$ systems, respectively, are clearly combinations of adsorbate FT motion and substrate phonon motion. These modes exhibit the large amplitude C-O wagging behavior characteristic of frustrated 


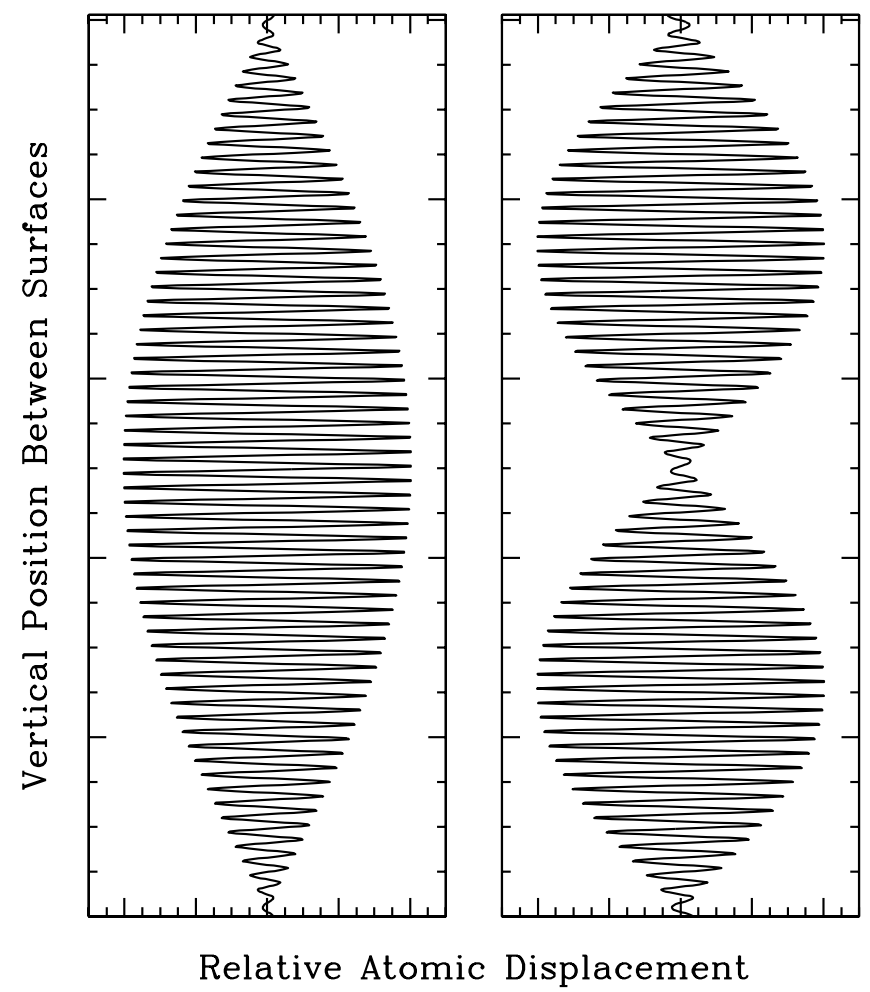

FIG. 3. Atomic displacement pattern for the first two modes below the band gap for a 2,000-layer, bare (6,2) superlattice substrate. The vertical axis denotes the position of a layer of atoms relative to the surface, and the horizontal axis denotes the relative displacement of each layer away from equilibrium. The top and bottom of the vertical axis are the positions of the top and bottom surface of the substrate.

translations. However, an appreciable amplitude of long-wavelength bulk-phonon motion persists throughout the substrate, so that the resonance states are not localized.

Comparing panels (b) and (c) of Fig. 2 shows that the substrate-phonon component of the mode has noticeably smaller amplitude for the $(6,2)$ system than for the $(8,0)$ system, indicating a smaller coupling strength between the adsorbate FT motion and the substrate phonons. We were able to infer the same result in the previous section by comparing both the DOS's and the FT-projected DOS's of the $(6,2)$ and $(8,0)$ systems.

To understand the source of this weaker coupling, we investigate the nature of the bulk modes in the enhanced spectral region below the band gap. Figure 3 illustrates the atomic displacement pattern for the first two modes below the band gap for a 2,000-layer, bare $(6,2)$ superlattice substrate. The vertical axis again denotes the position of a layer of atoms relative to the surface, but in this figure, unlike Fig. 2, all substrate layers are shown. These modes have a beat-like pattern, with the comparatively short-wavelength oscillations spanning about 16 (i.e., $2 N$ ) atomic layers per cycle and the long-wavelength oscillations exhibiting fixed-end standing-wave character.

The existence of these beat-like modes is tied to the formation of the band gap. For wavelengths around $2 N$ atomic layers in a period- $N$ superlattice, it becomes possible to form linear combinations of homogeneous-substrate modes that have maximal atomic displacement solely in either material- $A$ or material- $B$ regions of the superlattice. These linear combinations are stabilized by the coherence with the superlattice modulation, and they give rise to a band gap because of the inverse-square-root dependence of the frequency on the mass. The beat patterns form because the linear combinations superpose modes with similar spatial frequencies (i.e., wavelengths). There are many different ways to form linear combinations that are commensurate with the superlattice modulation, each one distinguished by the number of nodes in the resulting envelope waveform. This is the source of the spectral enhancement below and above the band gap.

For the $(6,2)$ system, the adsorbate FT motion is in resonance with these beat-like modes. However, since these modes have very small amplitude at the surface of the superlattice, the coupling between them and the FT mode is 


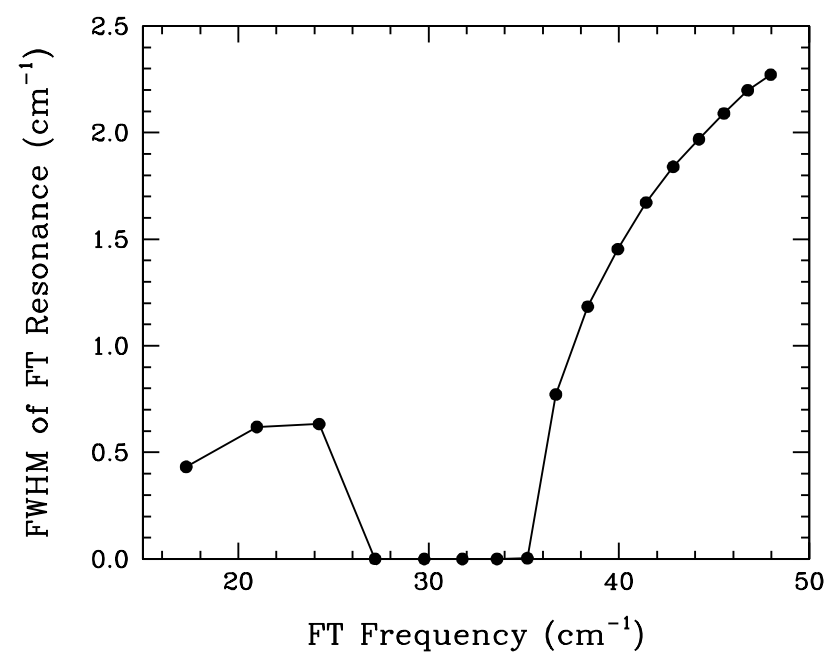

FIG. 4. Dependence of the FT resonance width on the FT frequency for the $(6,2)$ superlattice.

very weak. Therefore, despite the large density of bulk modes available for coupling, the resulting resonance peak remains very narrow, and the FT-mode remains long-lived.

To elucidate more fully the effect of the envelope function of the beat-like modes on FT vibrational relaxation, we examine how the width of the FT resonance varies with FT frequency, for a given superlattice substrate. We are able to accomplish this by varying the value of the force constant, $K_{a s}$, coupling the adsorbates to the substrate, [13] since the FT frequency is a smooth, monotonically increasing function of $K_{a s}$. Figure 1 displays the computed dependence of the resonance width on the FT frequency for the $(6,2)$ superlattice. [14] As expected, the resonance width is zero for FT frequencies in the superlattice-induced bulk band gap. The weak coupling of the FT to the beat-like modes near the gap edges is seen as a rapid decrease in the resonance width when approaching the band-gap region from either side. Away from the bulk band gap and its adjacent spectrally enhanced regions, the resonance width is a monotonic, more gently increasing function of the FT frequency.

One concern regarding the analysis in this paper is that superlattices with very short modulation periods are required. As a result, the interfaces between the $A$ and $B$ layers must be sharp on the scale of one or two atomic layers. This sharpness may be difficult to achieve for metallic superlattices due to interfacial diffusion or miscibility. A potential solution to this concern would be to use a semiconductor superlattice for the substrate. These materials can be grown with very sharp interfaces, and therefore may accommodate the short modulation periods that are required for the application described in this paper. In order to restore the chemical environment of the adsorbate-metal interface, one could first deposit several layers of metal (e.g., copper) on the surface of the superlattice, and then form the molecular overlayer on the supported metal film.

\section{CONCLUSIONS}

One of the most important goals in surface science is to be able to control dynamical processes taking place at a surface. We have proposed substrate patterning as a means to control the relaxation dynamics of low-frequency frustrated-translational vibrations of an adsorbate overlayer. This proposal emerges from our recent work demonstrating that the dominant relaxation mechanism for these modes is resonant emission of phonons into the substrate. Specifically, we have considered short-period superlattices as candidate substrates, because the mass modulation of a superlattice causes gaps to open in the phonon density of states. Varying the superlattice composition allows one to control the size and placement of these band gaps, thereby controllably modifying the coupling between the adsorbate overlayer and the substrate. We view the successful experimental demonstration of the behavior predicted here as a stringent test of our theory of resonant adsorbate-substrate coupling. Moreover it would establish substrate patterning as an important tool in surface dynamical research. 


\section{ACKNOWLEDGMENTS}

Financial support for this research was provided by the National Science Foundation under Grant No. DMR 9702514, the Air Force Office of Scientific Research, Air Force Materiel Command, USAF, under Grant No. F49620-00-10170, and the University of Georgia Research Foundation. SPL acknowledges support of the Donors of The Petroleum Research Fund, administered by the American Chemical Society. AMR acknowledges support of the Alfred P. Sloan Foundation.

[1] B. N. J. Persson, Chem. Phys. Lett. 149, 278 (1988).

[2] S. P. Lewis and A. M. Rappe, Phys. Rev. Lett. 77, 5241 (1996).

[3] S. P. Lewis, M. V. Pykhtin, E. J. Mele, and A. M. Rappe, J. Chem. Phys. 108, 1157 (1998).

[4] M. V. Pykhtin, S. P. Lewis, E. J. Mele, and A. M. Rappe, Phys. Rev. Lett. 81, 5940 (1999).

[5] S. P. Lewis and A. M. Rappe, J. Chem. Phys. 110, 4619 (1999).

[6] Adsorbate vibrational relaxation via phonon emission was first considered theoretically by B. N. J. Persson and R. Ryberg, Phys. Rev. B 32, 3586 (1985), and by B. Hall, D. L. Mills, and J. E. Black, Phys. Rev. B 32, 4932 (1985).

[7] A. Kueny, M. Grimsditch, K. Miyano, I Banerjee, C. M. Falco, and I. K. Schuller, Phys. Rev. Lett. 48, 166 (1982).

[8] A. Kueny and M. Grimsditch, Phys. Rev. B 26, 4699 (1982).

[9] R. E. Camley, B. Djafari-Rouhani, L. Dobrzynski, and A. A. Maradudin, Phys. Rev. B 27, 7318 (1983).

[10] G. Carlotti, D. Fioretto, L. Palmieri, G. Socino, L. Verdini, H. Xia, A. Hu, and X. K. Zhang, Phys. Rev. B 46, 12777 (1992).

[11] E. H. El Boutouti, B. Djafari-Rouhani, A. Akjouj, and L. Dobrzynski, Phys. Rev. B 54, 14728 (1996).

[12] F. Hofmann and J. P. Toennies, Chem. Rev. 96, 1307 (1996).

[13] The variation of this force constant has to be done in a manner that preserves the acoustic sum rule (i.e., acoustic modes go to zero frequency as wavelength becomes infinite). Thus, if $K_{i j}$ is the force constant in question, then incrementing $K_{i j}$ by an amount $\Delta$ requires decrementing $K_{i i}$ and $K_{j j}$ by the same amount.

[14] Figure 4 corresponds to the case where the superlattice substrate terminates on the more massive material, $B$. This choice has the effect of suppressing the intra-gap surface state. The alternate choice of terminating on an $A$-type layer leads to a more complicated picture involving an avoided crossing of the FT and surface states as a function of $K_{a s}$. 